\title{
ГАУССОВЕ НАБЛИЖЕННЯ В ОПТИМІЗАЦІЙНІЙ ЗАДАЧІ МОДЕЛІ ГРИ У МЕНШОСТІ
}

\section{В.С. ЯНІШЕВСЬКИЙ}

УДК 541.183 (C) 2011
Дрогобицький державний педагогічний університет ім. I. Франка

(Вул. I. Франка, 24, Дрогобич 82100; e-mail: yanishev@ukr. net)
Методами статистичної фізики досліджено оптимізаційну задачу у відомій моделі гри у меншості. Оптимізаційну задачу зведено до вивчення основного стану реплічного гамільтоніана з випадковими параметрами деякої ефективної системи 3 неперервним спіном. Використовуючи ідеї центральних граничних теорем теорії ймовірностей, отримано представлення для функції розподілу параметрів гамільтоніана і виконано перехід до гауссового розподілу у випадку великих $P$. Застосовуючи наближення $1 R S B$ та $2 R S B$ в методі реплік, отримано залежність мінімуму досліджуваної величини від параметра $\alpha$. Показано, що в області застосовності запропонований метод дає менші значення мінімуму, ніж в оригінальних роботах.

\section{1. Вступ}

Як відомо [1-3], за допомогою методів і підходів статистичної фізики невпорядкованих систем отримано низку важливих результатів у класичних оптимізаційних задачах дослідження операцій, моделях нейронних мереж і ряду інших систем. Останніми роками розвивається напрям еконофізики [4], де методи і підходи статистичної фізики використовуються в моделюванні економічних систем. Відомий ряд моделей, що були запропоновані фізиками для опису фінансових і фондових ринків $[4,5]$. Звернемо увагу на модель гри у меншості, що вивчалась в [6-12]. Гра у меншості охоплює різносторонні дослідження, проте в даній роботі розглянемо оптимізаційну задачу, що виникає в даній моделі. Основи моделі досить детально висвітлені в літературі (див., наприклад, [11]), тому наведемо стисло її суть. В моделі гри у меншості ринок моделюється ігровою взаємодією $N$ агентів. В кожен момент часу $t i$-й агент $(i=1, \ldots, N)$ здійснює дію $a_{i}(t)=1$ (купівля) або $a_{i}(t)=-1$ (продаж). Ви- граш $i$-го агента $u_{i}(t)$ із врахуванням дій всіх агентів визначається за формулою

$u_{i}(t)=-a_{i}(t) A(t), \quad$ де $\quad A(t)=\sum_{i=1}^{N} a_{i}(t)$.

Формула (1) моделює взаємодію між агентами на ринку через глобальну величину $A(t)$. Очевидно, що в кожен момент часу всіх агентів ринку можна розділити на дві групи за вибраною дією (купівля чи продаж). 3 визначення (1) випливає правило меншості - виграє той, хто перебуває в меншості. Виграшну дію агента, який перебуває у меншості можна записати у вигляді $a_{i}(t)=-\operatorname{sign}(A(t))$, а його виграш становить $|A(t)|$. Відповідно дія агента більшості рівна $a_{i}(t)=\operatorname{sign}(A(t))$, а його програш $\left.-|A(t)|\right)$. Загальний виграш усіх агентів становить $\sum_{i} u_{i}=-A^{2}$ і завжди від'ємний.

Всі агенти мають доступ до спільної інформації, яку в момент часу $t$ описують цілим числом $\mu(t)=1, \ldots, P$. Оскільки поведінка агентів впливає на ринок, то цю дію позначають через $A^{\mu(t)}(t)$. Як правило, існує $2^{P}$ стратегій, проте вважається, що кожен агент вибирає із загальної кількості лише $S$. Дію $i$-го агента, якщо він дотримується стратегії $s$ i використовує інформацію $\mu(t)$ позначимо $a_{s, i}^{\mu}$ (в стаціонарному випадку часову змінну $t$ опускаємо). Вважається, що число $P$ досить велике, того ж самого порядку, що й $N$, а величина $\alpha=P / N$ скінченна для $N \rightarrow \infty$ і $P \rightarrow \infty$. Значення $\mu$ описується певним розподілом $\rho^{\mu}$, незалежно для кожного часового кроку (далі вважаємо, що $\rho^{\mu}=1 / P$ ). В моделі задається також, що для кожного часового кроку дії агентів $a_{s, i}^{\mu} \mathrm{\epsilon}$ 
незалежними випадковими подіями з ймовірностями:

$P\left(a_{s, i}^{\mu}=+1\right)=P\left(a_{s, i}^{\mu}=-1\right)=\frac{1}{2}$,

$i=1, \ldots, N, s=1, \ldots, S, \mu=1, \ldots, P$.

Далі для аналізу гри введемо змішані стратегії $\pi_{s, i}$ (див., наприклад, [13]), що визначають ймовірності, з якими $i$-й агент застосовує дану стратегію (виконується умова $\left.\sum_{s} \pi_{s, i}=1\right)$. Множина змінних $\pi_{s, i}$ визначає фазовий простір моделі [11]. У фазовому просторі визначають середні значення величин:

$$
\left\langle A^{\mu}\right\rangle=\sum_{i=1}^{N} \sum_{s=1}^{S} \pi_{s, i} a_{s, i}^{\mu} .
$$

У роботах [9-11] для опису колективних властивостей моделі введена величина:

$$
H=\sum_{\mu=1}^{P} \rho^{\mu}\left(\sum_{i=1}^{N} \sum_{s=1}^{S} \pi_{s, i} a_{s, i}^{\mu}\right)^{2},
$$

що визначає флуктуації (2). Встановлено також [11], що (3) володіє властивостями гамільтоніана системи. У випадку, якщо $H=0$, то гра симетрична, тобто $\left\langle A^{\mu}\right\rangle=0$ для $\forall \mu(2)$ i, згідно з сказаним вище, середній виграш дорівнює нулю. Якщо ж $H>0$, то гра асиметрична (порушена симетрія між діями агентів продаж і купівля), отже існує $\mu$, для якого $\left\langle A^{\mu}\right\rangle \neq 0$ і має місце виграшна стратегія.

У наведених вище роботах вивчався мінімум (3) на множині змінних $\left\{\pi_{s, i}\right\}$. Дослідження мінімуму зводиться до обчислення "вільної енергії" системи, що описується гамільтоніаном (3) для "нульової температури", або для оберненої температури $\beta \rightarrow \infty$. Оскільки (3) містить залежність від дій гравців $a_{s, i}^{\mu}$, то для отримання змістовних величин виконувалось усереднення за всіма можливими діями агентів $a_{s, i}^{\mu}$. $\mathrm{V}$ результаті виникає задача теорії невпорядкованих систем із "замороженим" непорядком, що розв'язується в методі реплік. У роботах [6-12] для мінімуму (3) отримано асимптотично точний розв'язок для $N, P \rightarrow \infty$ і $P / N=\alpha \sim 1$. Зазначений розв'язок описує фазовий перехід з порушенням симетрії в точці $\alpha_{c} \approx 0,3374$. Тобто, для $\alpha \leqslant \alpha_{c}$ величина $H=0$ і для $\alpha>\alpha_{c}-H>0$. Оскільки параметр $\alpha$ пов'язаний з величиною $P$, що є мірою інформації, то зазначений розв'язок інтерпретують також як вплив інформації на ринок. А саме, у відсутності інформації має місце повна рівновага на ринку, наявність інформації приводить до порушення рівноваги на ринку, тобто існує виграшна стратегія.

При отриманні асимптотичного розв'язку в роботах [9-11] використовується розклад за параметром $\sim \beta / P$, який вважається малим параметром для великих значень $P$. Власне використовується наближена рівність:

$\cos \left(\sqrt{\frac{\beta}{P}} x\right) \simeq \exp \left(-\frac{\beta}{2 P} x^{2}\right)$.

У роботі [14] було звернуто увагу на те, що наближення (4) справедливе для скінченних значень $\beta$, проте не може бути обцрунтовано для $\beta \rightarrow \infty$. Додатково можна вказати на протиріччя, що містить вказаний розв'язок. Зокрема, для отриманого розв'язку величина $\chi=\beta(Q-q) / \alpha$ розбігається для $\alpha \rightarrow \alpha_{c}$. Розбіжність, очевидно, пов'язана з тим, що для $\beta \rightarrow \infty$ $Q \neq q(0 \leq Q, q \leq 1)$. В той же час, у вихідних виразах при застосуванні наближення (4), величина $\beta \sum_{i, s} \pi_{s, i} \pi_{s, i} \sim \chi$ вважається обмеженою.

У зв'язку з цим, в роботі [14] було розглянуто спосіб побудови асимптотичного розв'язку, що грунтується на ідеях граничних теорем теорії ймовірностей [17]. Зокрема, для суми випадкових величин вигляду $\frac{1}{\sqrt{P}} \sum_{\mu}(\ldots)$ в границі $P \rightarrow \infty$ було здійснено перехід до гауссових змінних (розподілених за нормальним законом). У результаті пошук мінімуму (3) зводиться до вивчення основного стану гамільтоніана із параметрами, розподіленими за гауссовим законом. Розрахунки, виконані в наближенні симетричних реплік, виявили інші залежності (3) від параметра $\alpha$ порівняно із даними літературних джерел [9-11].

$\mathrm{У}$ даній роботі побудовано функцію розподілу (характеристичну функцію) параметрів гамільтоніана i більш послідовно виконано граничний перехід до гауссового наближення. Виконано дослідження основного стану гамільтоніана із порушенням реплічної симетрії на один $(1 R S B)$ і два кроки $(2 R S B)[15]$.

\section{2. Гауссове наближення}

Як було зазначено, задача полягає у визначенні мінімуму гамільтоніана $H$ на множині змінних $\left\{\pi_{s, i}\right\} .3$ цією метою визначимо статистичну суму системи

$Z(\beta)=\operatorname{Sp}_{\pi} \exp (-\beta H(\pi))$,

де $\beta$ - "обернена температура", $\mathrm{Sp}_{\pi}$ позначає інтегрування за змінними $\pi_{s, i} \in[0,1]$ із врахуванням умови 
$\sum_{s} \pi_{s, i}=1$ для кожного $i=1, \ldots, N$ :

$\operatorname{Sp}_{\pi}(\ldots)=\int_{0}^{1} \prod_{s, i} d \pi_{s, i}(\ldots)$.

Позначення $H(\pi)$ - вказує на залежність від ймовірностей змішаних стратегій $\left\{\pi_{s, i}\right\}$. Як і при дослідженні основного стану фізичних систем (див., наприклад, [16]), визначення мінімуму можна звести до вивчення основного стану гамільтоніана при нулі “температури". В результаті отримуємо співвідношення

$$
\min _{\pi} H(\pi)=-\lim _{\beta \rightarrow \infty} \frac{1}{\beta} \ln Z(\beta) .
$$

Після усереднення (6) за всіма можливими діями гравців $a_{s, i}^{\mu}$ одержимо

$\mathcal{H}=\left\langle\min _{\pi} H(\pi)\right\rangle_{a}=-\lim _{\beta \rightarrow \infty} \frac{1}{\beta}\langle\ln Z(\beta)\rangle_{a}$.

Для спрощення викладок розглянемо випадок двох станів $S=2$ [11] і представимо (3) у формі гамільтоніана, заданого на множині змінних $\pi_{i}$ з випадковими параметрами [14]. Параметри гамільтоніана є сумою (за індексом $\mu$ ) незалежних випадкових величин. У випадку великих значень $P \sim N$, згідно з граничними теоремами теорії ймовірностей [17], параметри випадкові величини, що наближено задаються гауссовим розподілом. Отже, від усереднення статсуми (7) за розподілами величин $a_{s, i}^{\mu}$ перейдемо до усереднення за гауссовими розподілами параметрів гамільтоніана. Після очевидних перетворень гамільтоніан (3) запишемо у вигляді

$H=\hat{C}_{0}+\sum_{i} \hat{d}_{i} \pi_{i}^{2}+\sum_{i<j} \hat{J}_{i j} \pi_{i} \pi_{j}+\sum_{i \neq j} \hat{k}_{i j} \pi_{i}$

де введено позначення:

$$
\begin{aligned}
& \hat{C}_{0}=\frac{1}{4 P} \sum_{\mu}\left(\sum_{i} a_{i+}^{\mu}\right)^{2}, \quad \hat{d}_{i}=\frac{1}{4 P} \sum_{\mu}\left(a_{i-}^{\mu}\right)^{2}, \\
& \hat{J}_{i j}=\frac{1}{2 P} \sum_{\mu} a_{i-}^{\mu} a_{j-}^{\mu}, \quad \hat{k}_{i j}=\frac{1}{2 P} \sum_{\mu} a_{i-}^{\mu} a_{j+}^{\mu}, \\
& a_{i \pm}^{\mu}=a_{1, i}^{\mu} \pm a_{2, i}^{\mu} .
\end{aligned}
$$

Після підстановки (8) в (7) легко бачити, що $\left\langle\hat{C}_{0}\right\rangle_{a}=N / 2$. Решту доданків у (8) мають структуру гамільтоніана системи із неперервним спіном $\left(\pi_{i} \in[-1,1], i=1, \ldots, N\right)$. Зауважимо, що вираз (8) не містить жодних наближень і є лише іншою формою запису гамільтоніана (3). Перейдемо до нормованих параметрів $\left\{\tilde{d}_{i}, \tilde{J}_{i j}, \tilde{k}_{i j}\right\}$, середні значення і дисперсії яких дорівнюють 0 і 1 відповідно. Отже, маємо

$$
\begin{aligned}
& H=\frac{1}{2} \sum_{i}\left(\frac{\tilde{d}_{i}}{\sqrt{P}}+1\right) \pi_{i}^{2}+\frac{1}{\sqrt{P}} \sum_{i<j} \tilde{J}_{i j} \pi_{i} \pi_{j}+ \\
& +\frac{1}{\sqrt{P}} \sum_{i \neq j} \tilde{k}_{i j} \pi_{i} .
\end{aligned}
$$

Нормовані параметри виражаються через попередні (9) співвідношеннями

$\tilde{d}_{i}=\sqrt{P}\left(2 \hat{d}_{i}-1\right), \tilde{J}_{i j}=\sqrt{P} \hat{J}_{i j}, \tilde{k}_{i j}=\sqrt{P} \hat{k}_{i j}$.

Введемо функцію розподілу $\Psi(d, J, k)$ параметрів (11) і представимо співвідношення для мінімуму (7) у вигляді

$$
\mathcal{H}=\frac{N}{2}-\lim _{\beta \rightarrow \infty} \frac{1}{\beta}\langle\ln \mathcal{Z}(\beta)\rangle_{\Psi},
$$

де

$$
\mathcal{Z}(\beta)=\int_{-1}^{1} \prod_{i} \frac{d \pi_{i}}{2} \exp (-\beta H) .
$$

Розрахунок функції розподілу $\Psi(d, J, k)$ (ï характеристичної функції) наведено в додатку А, де показано, що в головному порядку за параметром $1 / \sqrt{P}$, функція $\Psi(d, J, k)$ прямує до гауссової функції розподілу (А.11). Використовуючи гауссову функцію розподілу (А.11), усереднення в (12) в методі реплік виконується в замкнутому вигляді і в результаті отримуємо асимптотично точний розв'язок.

\section{3. Метод реплік}

В методі реплік $[3,15]$ покладемо:

$$
\langle\ln \mathcal{Z}(\beta)\rangle_{\Psi}=\lim _{n \rightarrow 0} \frac{1}{n} \ln \left\langle\mathcal{Z}(\beta)^{n}\right\rangle_{\Psi}
$$

Як відомо, усереднення в (13) виконується для цілих $n$, після чого здійснюється аналітичне продовження для дійсних $n$. В результаті вираз для мінімуму (7) запишемо у вигляді

$\mathcal{H}=\frac{N}{2}-\lim _{\beta \rightarrow \infty} \frac{1}{\beta} \lim _{n \rightarrow 0} \frac{1}{n} \ln \left\langle\mathcal{Z}(\beta)^{n}\right\rangle_{\Psi}$. 
Статсума системи $n$ реплік визначається виразом

$\mathcal{Z}(\beta)^{n}=\operatorname{Sp}_{\pi} \exp \left(-\beta H^{(n)}\right)$.

У формулі (15) позначено гамільтоніан $n$ реплік:

$H^{(n)}=\frac{1}{2} \sum_{i, a}\left(\frac{d_{i}}{\sqrt{P}}+1\right) \pi_{i}^{a 2}+\sum_{i<j, a} \frac{J_{i j}}{\sqrt{P}} \pi_{i}^{a} \pi_{j}^{a}+$

$+\frac{1}{\sqrt{P}} \sum_{i \neq j, a} k_{i j} \pi_{i}^{a}$

де індекс $a=1, \ldots, n$ нумерує репліки і введено позначення:

$\operatorname{Sp}_{\pi}(\ldots)=\int_{-1}^{1} \prod_{i, a} \frac{d \pi_{i}^{a}}{2}(\ldots)$.

Після усереднення статсуми $n$ реплік (15) за гауссовим розподілом (А.11) отримаємо

$$
\left\langle\mathcal{Z}(\beta)^{n}\right\rangle_{\Psi}=\operatorname{Sp}_{\pi} \exp \left(-\beta H_{0}^{(n)}-\beta H_{\text {int }}^{(n)}\right),
$$

де введено позначення:

$$
\begin{aligned}
& H_{0}^{(n)}=\frac{1}{2} \sum_{i, a} \pi_{i}^{a 2} \\
& H_{\mathrm{int}}^{(n)}=-\frac{\beta}{8 P} \sum_{i}\left(\sum_{a} \pi_{i}^{a 2}\right)^{2}-\frac{\beta N}{2 P} \sum_{i}\left(\sum_{a} \pi_{i}^{a}\right)^{2}- \\
& -\frac{\beta}{2 P} \sum_{i<j}\left(\sum_{a} \pi_{i}^{a} \pi_{j}^{a}\right)^{2} .
\end{aligned}
$$

Складова гамільтоніана $H_{\text {int }}^{(n)}$ описує ефективну взаємодію між репліками. Першим доданком в (19) можна знехтувати, оскільки його внесок $\sim N^{0}$. Подальші обчислення полягають в факторизації інтегралів за змінними частинок. Ввівши матрицю перекриття між репліками $\hat{Q}_{a b}=\frac{1}{\sqrt{N}} \sum_{i} \pi_{i}^{a} \pi_{i}^{b}$, запишемо $H_{\mathrm{int}}^{(n)} \mathrm{y}$ вигляді

$H_{\mathrm{int}}^{(n)}=-\frac{\beta N}{4 P} \sum_{a, b} \hat{Q}_{a b}^{2}-\frac{\beta N}{2 P} \sqrt{N} \sum_{a, b} \hat{Q}_{a b}$.

Виконуючи підстановку $P=\alpha N$ для $\beta \rightarrow \sqrt{\alpha} \beta$ отримуємо для мінімуму:

$\mathcal{H}=\frac{N}{2}-\lim _{\beta \rightarrow \infty} \frac{1}{\sqrt{\alpha} \beta} \lim _{n \rightarrow 0} \frac{1}{n} \ln \overline{\mathcal{Z}}(\beta)^{(n)}$.

Статсума $n$ реплік після зазначених перетворень визначається виразом

$\overline{\mathcal{Z}}(\beta)^{(n)}=\operatorname{Sp}_{\pi} \exp \left(-\beta \bar{H}_{0}^{(n)}-\beta \bar{H}_{\text {int }}^{(n)}\right)$,

де

$\bar{H}_{0}^{(n)}=\frac{\sqrt{\alpha}}{2} \sum_{i, a} \pi_{i}^{a 2}$,

$\bar{H}_{\mathrm{int}}^{(n)}=-\frac{\beta}{4} \sum_{a, b} \hat{Q}_{a b}^{2}-\frac{\beta}{2} \sqrt{N} \sum_{a, b} \hat{Q}_{a b}$.

Формула (23) визначає складові ефективного гамільтоніана після усереднення за гауссовим розподілом. Залежність від параметра $\alpha$ міститься лише в доданку $\bar{H}_{0}^{(n)}$, який можна, за аналогією зі спіновими системами, трактувати як одновузлову анізотропію. Із $(21)$ i (23) випливає також, що величина $\mathcal{H}$ монотонно залежить від $\alpha$. При зменшенні $\alpha$ завдяки множнику $1 / \sqrt{\alpha}$ друга складова в (21) зростає, що приводить до зменшення $\mathcal{H}$ і для деякого $\alpha_{0} \mathcal{H}$ стає рівним нулю. Таким чином, вважатимемо, що наближення (23) застосовне для $\alpha>\alpha_{0}$. За допомогою інтегрального перетворення

$e^{\frac{1}{2} x^{2}}=\frac{1}{\sqrt{2 \pi}} \int_{-\infty}^{\infty} d z e^{-\frac{1}{2} z^{2}} e^{z x}$

лінеаризуємо складову $\sim \hat{Q}_{a b}^{2}$ в $(22)$ і факторизуємо статсуму (22) за змінними частинок. У результаті отримаємо представлення статсуми у просторі реплічних змінних:

$\overline{\mathcal{Z}}(\beta)^{(n)}=L \int D Q \exp (N \Phi(Q))$,

де позначено:

$L=\left(\frac{\beta}{\sqrt{2}}\right)^{n^{2}}, D Q=\prod_{a, b} \frac{d Q_{a b}}{\sqrt{2 \pi}}$,

$\Phi(Q)=-\frac{\beta^{2}}{4} \sum_{a, b} Q_{a b}^{2}+\ln Z_{1}(Q)$,

$Z_{1}(Q)=\int_{-1}^{1} \prod_{a} \frac{d \pi_{a}}{2} \exp \left(\Phi_{1}(Q)\right)$

ISSN 2071-0194. Укр. фіз. журн. 2011. T. 56, №1 
$\Phi_{1}(Q)=-\beta \frac{\sqrt{\alpha}}{2} \sum_{a} \pi_{a}^{2}+\frac{\beta^{2}}{2} \sum_{a, b} Q_{a b} \pi_{a} \pi_{b}+\frac{\beta^{2}}{2}\left(\sum_{a} \pi_{a}\right)^{2}$

В границі $N \rightarrow \infty$ інтеграли в (24) обчислюємо методом Лапласа і отримуємо

$\overline{\mathcal{Z}}(\beta)^{(n)}=\exp (N \Phi(\bar{Q}))$,

де $\bar{Q}$ - екстремальне значення, яке знаходиться з рівнянь стаціонарності:

$\frac{\partial \Phi(Q)}{\partial Q_{a b}}=0 \rightarrow \bar{Q}_{a b}=\left\langle\pi_{a} \pi_{b}\right\rangle_{\pi}$

Зміст усереднення в (27) визначається виразом

$$
\langle(\ldots)\rangle_{\pi}=\frac{1}{Z_{1}(\bar{Q})} \int_{-1}^{1} \prod_{a} \frac{d \pi_{a}}{2} \exp \left[\Phi_{1}(\bar{Q})\right](\ldots) .
$$

У результаті для мінімуму величини $\mathcal{H}$ отримаємо співвідношення

$\mathcal{H}=\frac{N}{2}+\frac{N}{\sqrt{\alpha}} \lim _{\beta \rightarrow \infty} \frac{1}{\beta} \lim _{n \rightarrow 0} \frac{1}{n}\left(\frac{\beta^{2}}{4} \sum_{a, b} \bar{Q}_{a b}^{2}-\ln Z_{1}(\bar{Q})\right)$.

Очевидно, множник $L$ в (24) не дає внеску в границі $n \rightarrow 0$. Отже, формула (29) задає розв'язок задачі в методі реплік. Подальші обчислення полягають у конкретному виборі матриці перекриття $\hat{Q}$. В наближенні симетричних реплік $(R S)$, яке розглядалось в роботі [14], матриця $\hat{Q}$ задається у вигляді

$Q_{a b}= \begin{cases}Q, & a=b \\ q, & a \neq b\end{cases}$

Наближення з порушенням реплічної симетрії $1 R S B$ i $2 R S B$ розглянуто в додатках $\mathrm{B}$ i C.

\section{4. Розв'язок для $\boldsymbol{\beta} \rightarrow \infty$}

\section{1. Наближення $1 R S B$}

Загальні співвідношення наближення $1 R S B$ виписані в додатку В. Розглянемо границю $\mathcal{H}($ В.7) для $\beta \rightarrow \infty$. Введемо позначення для величин $\chi=\beta\left(Q-q_{1}\right)$ i $\chi_{1}=\beta\left(q_{1}-q\right)$. За аналогією зі спіновими системами, введені величини називають сприйнятливостями. Розглянемо спочатку випадок, що зазначені величини є скінченними для $\beta \rightarrow \infty$. Виконуючи послідовні перетворення змінної $z_{1} \rightarrow \sqrt{\beta} \sqrt{1+q} / \sqrt{\chi_{1}} z_{1}$ i $z_{1} \rightarrow z_{1}-z$, представимо величини $Z_{1}$ і $\tilde{Z}_{1}($ В.6) у вигляді

$Z_{1}=\frac{\sqrt{\beta} \sqrt{1+q}}{\sqrt{\chi_{1}}} \int_{-\infty}^{\infty} \frac{d z_{1}}{\sqrt{2 \pi}} \exp \left(-\beta \frac{1+q}{2 \chi_{1}}\left(z_{1}-z\right)^{2}\right) \tilde{Z}_{1}^{m}$

$\tilde{Z}_{1}=\int_{-1}^{1} \frac{d \pi}{2} \exp (\beta \phi(\pi))$

$\phi(\pi)=-\frac{\sqrt{\alpha}}{2} \pi^{2}+\frac{1}{2} \chi \pi^{2}+\sqrt{1+q} \pi z_{1}$.

Легко бачити, що для $\beta \rightarrow \infty$

$\frac{\sqrt{\beta} \sqrt{1+q}}{\sqrt{2 \pi \chi_{1}}} \exp \left(-\beta \frac{1+q}{2 \chi_{1}}\left(z_{1}-z\right)^{2}\right) \rightarrow \delta\left(z_{1}-z\right)$.

Звідси випливає, що при підстановці (31) у формули (В.7), (В.8), інтеграли за змінною $z_{1}$ обчислюються в замкнутому вигляді і ми отримаємо наближення $R S$ [14]. Очевидно, це є наслідком того, що величина $\chi_{1}=\beta\left(q_{1}-q\right)$ вважалась скінченною для $\beta \rightarrow \infty$. Інакше кажучи, у випадку $\beta \rightarrow \infty$ випливає, що $q_{1} \rightarrow q$ і відповідно наближення $1 R S B$ переходить в $R S$ (див. також зауваження в додатках В і C). Зазначимо, що на цю властивість наближення $1 R S B$ вказувалось також у роботах $[20,21]$, де їі спостерігали під час чисельних розрахунків.

Розв'язки відмінні від наближення $R S$ виникають, якщо задати залежність параметра у вигляді $m=$ $m_{0} / \beta$. Тоді величина $\Delta q=q_{1}-q \in$ скінченною для $\beta \rightarrow \infty$. Наступним кроком обчислюємо асимптотику величини $\tilde{Z}_{1}$ (В.6). Попередньо в формулах (В.7), (В.8) і (В.12) виконаємо послідовно заміну змінних:

$z \rightarrow \frac{z}{1+q}, z_{1} \rightarrow \frac{z_{1}}{\Delta q}, z_{1} \rightarrow z_{1}-z$.

У результаті для величини $\tilde{Z}_{1}$ отримаємо

$\tilde{Z}_{1}=\int_{-1}^{1} \frac{d \pi}{2} \exp (\beta \phi(\pi))$,

$\phi(\pi)=-\frac{\sqrt{\alpha}}{2} \pi^{2}+\frac{1}{2} \chi \pi^{2}+\pi z_{1}$

Для $\beta \rightarrow \infty$ інтеграл за змінною $\pi$ обчислюємо методом Лапласа. Максимум $\phi(\pi)$ досягається в точці

$\pi_{\max }= \begin{cases}-1, & \text { якщо } z_{1}<-z_{0}, \\ z_{1} / z_{0}, & \text { якщо }-z_{0}<z_{1}<z_{0}, \\ 1, & \text { якщо } z_{1}>z_{0},\end{cases}$ 


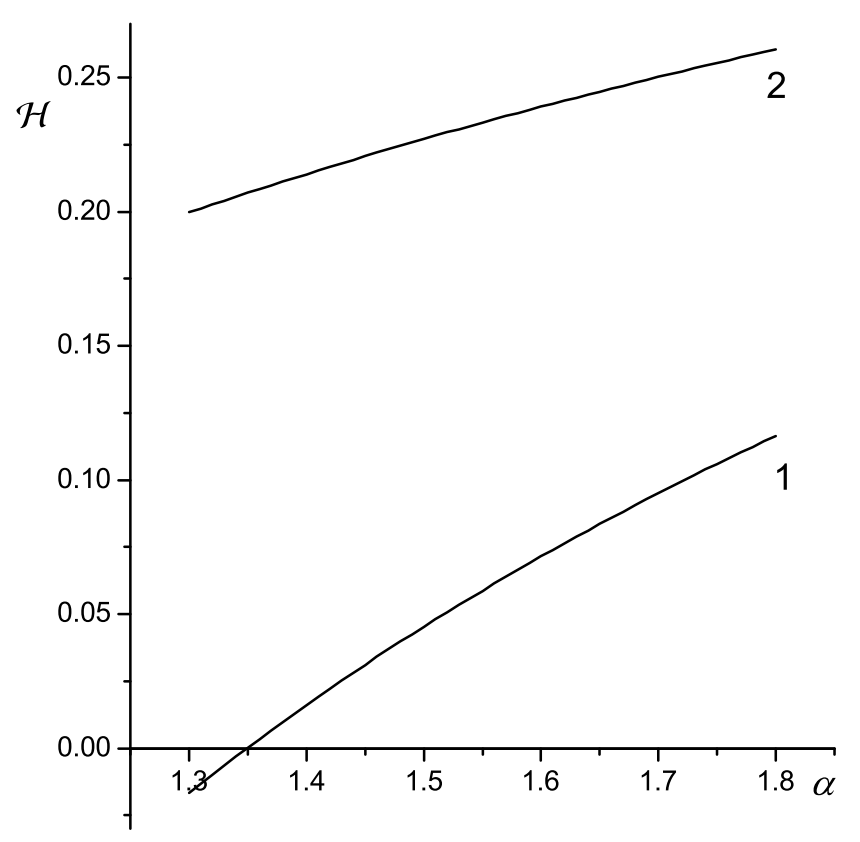

Залежність величини $\mathcal{H}$ від параметра $\alpha$ даної роботи (крива $1)$; крива перетинає вісь в точці $\alpha_{0} \approx 1,345$; фрагмент відповідної залежності за результатами роботи [11] (крива 2)

$z_{0}=\sqrt{\alpha}-\chi$

Відповідно для $\tilde{Z}_{1}$ і $Z_{1}$ отримаємо

$\tilde{Z}_{1} \simeq \exp \left(m_{0} \phi_{\max }\right), Z_{1} \simeq\left\langle\exp \left(m_{0} \phi_{\max }\right)\right\rangle_{z_{1}}$,

де

$\phi_{\max }= \begin{cases}-z_{0} / 2-z_{1}, & \text { якщо } z_{1}<-z_{0}, \\ z_{1}^{2} /\left(2 z_{0}\right), & \text { якщо }-z_{0}<z_{1}<z_{0}, \\ -z_{0} / 2+z_{1}, & \text { якщо } z_{1}>z_{0} .\end{cases}$

У результаті після нескладних алгебраїчних перетворень отримаємо в границі $\beta \rightarrow \infty$

$\mathcal{H} / N=\frac{1}{2}+\frac{1}{4 \sqrt{\alpha}}\left[2 \chi(\Delta q+q)+m_{0} \Delta q(\Delta q+2 q)\right]-$

$-\frac{1}{\sqrt{\alpha}} \frac{1}{m_{0}}\left\langle\ln \left\langle e^{m_{0} \phi_{\max }}\right\rangle_{z_{1}}\right\rangle_{z}$

На основі (В.8) і (В.12) отримуємо систему рівнянь для визначення параметрів $\chi, \Delta q, q, m_{0}$ :

$\chi=\frac{1}{\sqrt{1+q}}\left\langle\frac{\left\langle e^{m_{0} \phi_{\max }} \frac{d}{d z_{1}} \pi_{\max }\right\rangle_{z_{1}}}{\left\langle e^{m_{0} \phi_{\max }}\right\rangle_{z_{1}}}\right\rangle_{z}$,

$\Delta q+q=\left\langle\frac{\left\langle\pi_{\max }^{2} e^{m_{0} \phi_{\max }}\right\rangle_{z_{1}}}{\left\langle e^{m_{0} \phi_{\max }}\right\rangle_{z_{1}}}\right\rangle_{z}$,

$$
\begin{aligned}
& \Delta q=\left\langle\frac{\left\langle\pi_{\max } e^{m_{0} \phi_{\max }}\right\rangle_{z_{1}}^{2}}{\left\langle e^{m_{0} \phi_{\max }}\right\rangle_{z_{1}}^{2}}\right\rangle_{z}, \\
& \frac{1}{4} \Delta q(\Delta q+2 q)+\frac{1}{m_{0}^{2}}\left\langle\ln \left\langle e^{m_{0} \phi_{\max }}\right\rangle_{z_{1}}\right\rangle_{z}- \\
& -\frac{1}{m_{0}}\left\langle\frac{\left\langle\phi_{\max } e^{m_{0} \phi_{\max }}\right\rangle_{z_{1}}}{\left\langle e^{m_{0} \phi_{\max }}\right\rangle_{z_{1}}}\right\rangle_{z}=0 .
\end{aligned}
$$

Усереднення за змінними $z$ i $z_{1}$ визначаються формулами

$$
\begin{aligned}
& \langle\ldots\rangle_{z}=\int_{-\infty}^{\infty} \frac{d z}{\sqrt{2 \pi(1+q)}} \exp \left(-\frac{z^{2}}{2(1+q)}\right)(\ldots), \\
& \langle\ldots\rangle_{z_{1}}=\int_{-\infty}^{\infty} \frac{d z_{1}}{\sqrt{2 \pi \Delta q}} \exp \left(-\frac{\left(z_{1}-z\right)^{2}}{2 \Delta q}\right)(\ldots) .
\end{aligned}
$$

Систему рівнянь (36) досліджували чисельним методом. Для порівняння з результатами $R S[14]$ розглянемо залежність $\Delta q$ від $\alpha$. З'ясувалось, що відмінні від нуля значення $\Delta q$ спостерігаються в області параметра $\alpha \lesssim 1,32$, причому $\Delta q$ монотонно спадає до нуля із зростанням $\alpha$. Як зазначалось вище, в області значень параметра $\alpha \lesssim 1,32$ дане наближення не застосовне, оскільки $\mathcal{H}<0$. В свою чергу, для $\Delta q \rightarrow 0$ результати наближення $1 R S B$ збігаються із наближенням симетричних реплік, про що вказувалось в додатку В. Цю обставину можна проілюструвати також таким наглядним чином. Для $\Delta q \rightarrow 0$ функція розподілу змінної $z_{1}$ в (37) прямує до $\delta$-функції

$\frac{1}{\sqrt{2 \pi \Delta q}} \exp \left(-\frac{\left(z_{1}-z\right)^{2}}{2 \Delta q}\right) \rightarrow \delta\left(z_{1}-z\right)$

і інтегрування у формулах (35) i (36) за змінною $z_{1}$ виконуються в замкнутому вигляді і ми приходимо до наближення $R S$ [14]. Отже, для $\alpha \gtrsim 1,32$ результати наближень $1 R S B$ і $R S$ практично збігаються, при цьому $\mathcal{H}=0$ досягається для $\alpha_{0} \approx 1,345$ (див. рисунок, крива 1).

\section{2. Наближення $2 R S B$}

За аналогією із наближенням $1 R S B$, виконаємо заміни змінних $m_{1} \rightarrow m_{1} / \beta, m_{2} \rightarrow m_{2} / \beta$. Сприйнятливість $\chi=\beta\left(Q-q_{2}\right)$ є скінченною для $\beta \rightarrow \infty$. Визначимо змінні $\Delta q_{1}=q_{1}-q, \Delta q_{2}=q_{2}-q_{1}$ і виконаємо 
такі заміни у співвідношеннях наближення $2 R S B$ формули (С.4), (C.5), (C.6) i (C.8)):

$z \rightarrow \frac{z}{1+q}, z_{1} \rightarrow \frac{z_{1}}{\Delta q_{1}}, z_{2} \rightarrow \frac{z_{2}}{\Delta q_{2}}$,

$z_{1} \rightarrow z_{1}-z, z_{2} \rightarrow z_{2}-z_{1}$

Після зазначених перетворень отримаємо для мінімуму $\mathcal{H}$ вираз

$\mathcal{H} / N=\frac{1}{2}+\frac{1}{4 \sqrt{\alpha}}\left[2 \chi\left(\Delta q_{1}+\Delta q_{2}+q\right)+\right.$

$\left.+m_{2} \Delta q_{2}\left(2 \Delta q_{1}+\Delta q_{2}+q\right)+m_{1} \Delta q_{1}\left(\Delta q_{1}+2 q\right)\right]-$

$-\frac{1}{\sqrt{\alpha}} \frac{1}{m_{1}}\left\langle\ln Z_{1}\right\rangle_{z}$

Формули (С.5) набувають вигляду

$Z_{1}=\left\langle\left\langle\tilde{Z}_{2}^{m_{2}}\right\rangle_{z_{2}}^{\frac{m_{1}}{m_{2}}}\right\rangle_{z_{1}}, \tilde{Z}_{2}=\int_{-1}^{1} \frac{d \pi}{2} \exp (\beta \phi(\pi))$

$\phi(\pi)=-\frac{\sqrt{\alpha}}{2} \pi^{2}+\frac{1}{2} \chi \pi^{2}+\pi z_{2}$

При цьому усереднення за змінними $z_{1}, z_{2}$ здійснюються з гауссовими функціями

$$
\begin{aligned}
\langle\ldots\rangle_{z_{2}} & =\int_{-\infty}^{\infty} \frac{d z_{2}}{\sqrt{2 \pi \Delta q_{2}}} \exp \left(-\frac{\left(z_{2}-z_{1}\right)^{2}}{2 \Delta q_{2}}\right)(\ldots), \\
\langle\ldots\rangle_{z_{1}} & =\int_{-\infty}^{\infty} \frac{d z_{1}}{\sqrt{2 \pi \Delta q_{1}}} \exp \left(-\frac{\left(z_{1}-z\right)^{2}}{2 \Delta q_{1}}\right)(\ldots),
\end{aligned}
$$

а за змінною $z$ згідно 3 першою формулою (37). Подальша схема розрахунків така ж, що і у випадку $1 R S B$. Спочатку обчислюємо асимптотику інтеграла за змінною $\pi$ (величина $\tilde{Z}_{2}$ в $\left.(39)\right)$. У результаті отримаємо

$$
Z_{1} \simeq\left\langle\left\langle\exp \left(m_{2} \phi_{\max }\right)\right\rangle_{z_{2}}^{\frac{m_{1}}{m_{2}}}\right\rangle_{z_{1}}, \quad \tilde{Z}_{2} \simeq\left\langle\exp \left(m_{2} \phi_{\max }\right)\right\rangle_{z_{2}} .
$$

Із порівняння формул (32) і (39) легко бачити, що $\phi_{\max }$ задається тією ж формулою, що і в $(34)$, лише iз заміною $z_{1} \rightarrow z_{2}$. При цьому $\pi_{\max }$ також визначається формулою (33) із аналогічною заміною $z_{1} \rightarrow z_{2}$.
У підсумку на основі рівнянь (С.6) і (С.8) в границі $\beta \rightarrow \infty$ отримуємо систему рівнянь для параметрів $\chi, \Delta q_{2}, \Delta q_{1}, q, m_{1}, m_{2}$ :

$\chi=\frac{1}{\sqrt{1+q}}\left\langle\frac{1}{Z_{1}}\left\langle\left\langle e^{m_{2} \phi_{\max }} \frac{d \pi_{\max }}{d z_{2}}\right\rangle_{z_{2}}\left\langle e^{m_{2} \phi_{\max }}\right\rangle_{z_{2}}^{\frac{m_{1}}{m_{2}}-1}\right\rangle_{z_{1}}\right\rangle_{z}$,

$\Delta q_{2}+\Delta q_{1}+q=$

$=\left\langle\frac{1}{Z_{1}}\left\langle\left\langle\pi_{\max }^{2} e^{m_{2} \phi_{\max }}\right\rangle_{z_{2}}\left\langle e^{m_{2} \phi_{\max }}\right\rangle_{z_{2}}^{\frac{m_{1}}{m_{2}}-1}\right\rangle_{z_{1}}\right\rangle_{z}$

$\Delta q_{1}+q=\left\langle\frac{1}{Z_{1}}\left\langle\left\langle\pi_{\max } e^{m_{2} \phi_{\max }}\right\rangle_{z_{2}}^{2}\left\langle e^{m_{2} \phi_{\max }}\right\rangle_{z_{2}}^{\frac{m_{1}}{m_{2}}-2}\right\rangle_{z_{1}}\right\rangle_{z}$,

$q=\left\langle\left\langle\left(\frac{1}{Z_{1}}\left\langle\left\langle\pi_{\max } e^{m_{2} \phi_{\max }}\right\rangle_{z_{2}}\left\langle e^{m_{2} \phi_{\max }}\right\rangle_{z_{2}}^{\frac{m_{1}}{m_{2}}-1}\right\rangle_{z_{1}}\right)^{2}\right\rangle_{z}\right.$

$\frac{1}{4} \Delta q_{1}\left(\Delta q_{1}+2 q\right)+\frac{1}{m_{1}^{2}}\left\langle\ln \left(Z_{1}\right)\right\rangle_{z}-$

$-\frac{1}{m_{1} m_{2}}\left\langle\frac{1}{Z_{1}}\left\langle\left\langle e^{m_{2} \phi_{\max }}\right\rangle_{z_{2}}^{\frac{m_{1}}{m_{2}}} \ln \left\langle e^{m_{2} \phi_{\max }}\right\rangle_{z_{2}}\right\rangle_{z_{1}}\right\rangle_{z}=0$

$\frac{1}{4} \Delta q_{2}\left(\Delta q_{2}+2 \Delta q_{1}+2 q\right)+$

$+\frac{1}{m_{2}^{2}}\left\langle\frac{1}{Z_{1}}\left\langle\left\langle e^{m_{2} \phi_{\max }}\right\rangle_{z_{2}}^{\frac{m_{1}}{m_{2}}} \ln \left\langle e^{m_{2} \phi_{\max }}\right\rangle_{z_{2}}\right\rangle_{z_{1}}\right\rangle_{z}-$

$-\frac{1}{m_{2}}\left\langle\frac{1}{Z_{1}}\left\langle\left\langle e^{m_{2} \phi_{\max }}\right\rangle_{z_{2}}^{\frac{m_{1}}{m_{2}}-1}\left\langle e^{m_{2} \phi_{\max }} \phi_{\max }\right\rangle_{z_{2}}\right\rangle_{z_{1}}\right\rangle_{z}=0$.

Згідно з вихідними положеннями методу реплік [15] виконується також умова $m_{1} / m_{2}<1$.

У результаті чисельного розв'язку було виявлено, що параметр $\Delta q_{2}$ монотонно спадає із зростанням $\alpha$ і перетворюється в 0 для $\alpha \approx 0,84$. Як зазначено в додатку С для $\Delta q_{2} \rightarrow 0$ наближення $2 R S B$ перейде у $1 R S B$. Це можна спостерігати також таким чином. Якщо в формулах (38), (40) здійснити підстановку

$\frac{1}{\sqrt{2 \pi \Delta q_{2}}} \exp \left(-\frac{\left(z_{2}-z_{1}\right)^{2}}{2 \Delta q_{2}}\right) \rightarrow \delta\left(z_{2}-z_{1}\right)$,

то інтегрування за змінною $z_{2}$ виконується в замкнутому вигляді і ми прийдемо до наближення $1 R S B$. 
Отже, для $\alpha \gtrsim 0,84$ результати збігаються з наближенням $1 R S B$, що досліджувалось в попередньому розділі.

Підсумовуючи, зазначимо, що в області застосовності гауссового наближення $\alpha \geqslant \alpha_{0}\left(\alpha_{0} \approx 1,345\right)$ підтверджуються результати наближення $R S$ [14] 3 незначним збільшенням $\alpha_{0}$. В результаті чисельних досліджень виявлено, що із зростанням параметра $\alpha$ наближення $2 R S B$ прямує до $1 R S B$, і наближення $1 R S B$ - до $R S$. Очевидно, така поведінка зумовлена домінуванням доданка $\bar{H}_{0}^{(n)}$ в ефективному реплічному гамільтоніані (23) для $\alpha \sim 1$.

У зв'язку з цим можна зробити також таке зауваження. Визначимо гауссові поля

$h_{i}=\frac{1}{\sqrt{N}} \sum_{j(\neq i)} k_{j i}$

з $\left\langle h_{i}\right\rangle=0,\left\langle h_{i}^{2}\right\rangle=1$. Тоді ефективний гамільтоніан (16) запишемо у еквівалентному вигляді

$H^{(n)} \approx \frac{1}{2} \sum_{i, a} \pi_{i}^{a 2}+\sum_{i<j, a} \frac{J_{i j}}{\sqrt{P}} \pi_{i}^{a} \pi_{j}^{a}+\sqrt{N / P} \sum_{i, a} h_{i} \pi_{i}^{a}$.

Після підстановки $P=\alpha N$ гамільтоніан (43) запишемо у вигляді

$H^{(n)} / \sqrt{\alpha} \approx \frac{\sqrt{\alpha}}{2} \sum_{i, a} \pi_{i}^{a} 2+\sum_{i<j, a} \frac{J_{i j}}{\sqrt{N}} \pi_{i}^{a} \pi_{j}^{a}+\sum_{i, a} h_{i} \pi_{i}^{a}$.

Гамільтоніан (44) можна вважати аналогом гамільтоніана Гатака-Шеррінгтона [22-24] для системи 3 неперервним спіном величини $\pi=1$ в гауссових полях $h_{i}$. Нам невідомі роботи, де вивчався основний стан гамільтоніана, що відповідає (44). Зазначимо, що в роботі [24] досліджувався основний стан системи з гамільтоніаном Гатака-Шеррінгтона для спіну $S=1$

$H=-\sum_{i, j} J_{i, j} S_{i} S_{j}+D \sum_{i} S_{i}^{2}$,

3 гауссовим розподілом взаємодій $J_{i, j} \quad\left(\left\langle J_{i, j}\right\rangle=0\right.$, $\left.\left\langle J_{i, j}^{2}\right\rangle=J / N\right)$. Цікаво зазначити, що крива залежності вільної енергії від параметра $D / J$ виявляє характерну монотонну залежність як і на рисунку (крива 1).

\section{5. Висновки}

В даній роботі розглянуто задачу мінімізації в моделі гри у меншості в гауссовому наближенні. В ньо- му від усереднення за дискретними змінними здійснено перехід до усереднення за неперервними гауссовими змінними в границі великих $N$ і $P$ і скінченного $\alpha=P / N$. При цьому оптимізаційна задача зведена до дослідження основного стану системи, гамільтоніан якої містить параметри, розподілені за гауссовим розподілом. У запропонованому підході, як зазначено у вступі, відсутній розклад за параметром $\beta / P$, що використовувався в оригінальних роботах. Оскільки гауссова функція розподілу параметрів гамільтоніана (А.11) є граничною для $P \gg 1$, то це накладає обмеження на застосовність результатів в області зміни параметра $\alpha$. В нашому випадку ми визначили область застосовності наближення значеннями параметра $\alpha$, для яких $\mathcal{H}>0$. Чисельні розв'язки показують, що в цій області достатньо обмежитись наближенням $R S$, що зумовлено структурою ефективного гамільтоніана (16). Підсумовуючи, зазначимо, що хоча неможливо провести порівняння результатів даної роботи і оригінальних робіт $[6,7,9-11]$ для значень $\alpha<1,345$, проте для $\alpha>1,345$ в гауссовому наближенні отримано менші значення величини $\mathcal{H}$ (див. рисунок). Уточнення методу, згідно з центральною граничною теоремою, може здійснюватись шляхом побудови асимптотичних розвинень для гауссової функції розподілу (див., наприклад, [17]), що є непростою обчислювальною задачею і вимагає подальших досліджень.

\section{ДОДАТки}

\section{А. Функція розподілу параметрів гамільтоніана}

Параметри гамільтоніана (10) залежать від змінних $a_{s, i}^{\mu}, s=(1,2)$. Перехід від усереднення деякої функції $\Phi(\tilde{d}, \tilde{J}, \tilde{k})$ параметрів (11) за змінними $a_{s, i}^{\mu}$ до усереднення 3 функцією розподілу цих параметрів виконаємо за допомогою співвідношення

$\langle\Phi(\tilde{d}, \tilde{J}, \tilde{k})\rangle_{a}=\int D d D J D k \Psi(d, J, k) \Phi(d, J, k)$,

де $\Psi(d, J, k)=\langle\delta(d-\tilde{d}) \delta(J-\tilde{J}) \delta(k-\tilde{k})\rangle_{a}$ - має зміст густини ймовірності розподілу змінних $\left\{d_{i}\right\},\left\{J_{i j}\right\},\left\{k_{i j}\right\}$. В формулі (А.1) введено позначення для добутку диференціалів:

$D d=\prod_{i} d d_{i}, D J=\prod_{i<j} d J_{i j}, D k=\prod_{i \neq j} d k_{i j}$

і $\delta$-функцій

$\delta(d-\tilde{d}) \delta(J-\tilde{J}) \delta(k-\tilde{k})=$

$=\prod_{i} \delta\left(d_{i}-\tilde{d}_{i}\right) \prod_{i<j} \delta\left(J_{i j}-\tilde{J}_{i j}\right) \prod_{i \neq j} \delta\left(k_{i j}-\tilde{k}_{i j}\right)$.

Стандартним чином (див., наприклад, [17]) визначимо характеристичну функцію

$\varphi(y, z, x)=\int D d D J D k D l \Psi(d, J, k) \times$ 
$\times \exp (i \mathbf{y d}+i \mathbf{z} \mathbf{J}+i \mathbf{x k})=\langle\exp (i \mathbf{y} \tilde{\mathbf{d}}+i \mathbf{z} \tilde{\mathbf{J}}+i \mathbf{x} \tilde{\mathbf{k}})\rangle_{a}$,

де позначено

$\mathbf{y d}=\sum_{i} y_{i} d_{i}, \mathbf{z} \mathbf{J}=\sum_{i<j} z_{i j} J_{i j}, \mathbf{x k}=\sum_{i \neq j} x_{i j} k_{i j}$.

В експоненті (А.2) зміст позначень заданий в формулах (А.3), лише замість величин $\left\{d_{i}\right\},\left\{J_{i j}\right\},\left\{k_{i j}\right\}$ потрібно підставити вирази (11). Характеристична функція (А.2) факторизується за індексом $\mu$. У результаті отримаємо

$\varphi(y, z, x)=\varphi_{1}(y, z, x)^{P}$,

$\varphi_{1}(y, z, x)=\left\langle\exp \left(i \mathbf{y} \tilde{\mathbf{d}}_{\mathbf{1}}+i \mathbf{z} \tilde{\mathbf{J}}_{\mathbf{1}}+i \mathbf{x} \tilde{\mathbf{k}}_{\mathbf{1}}\right\rangle_{a}\right.$.

Індекс 1 в (А.4) вказує, що береться один доданок із суми за $\mu$ у відповідних виразах, що визначають $\tilde{d}, \tilde{J}, \tilde{k}$. Зокрема,

$\mathbf{y} \tilde{\mathbf{d}}_{\mathbf{1}}=-\sum_{i} y_{i} a_{1, i} a_{2, i} / \sqrt{P}, \mathbf{z} \tilde{\mathbf{J}}_{\mathbf{1}}+\mathbf{x} \tilde{\mathbf{k}}_{\mathbf{1}}=\sum_{i} a_{i-} B_{i}$,

$B_{i}=\frac{1}{2 \sqrt{P}} \sum_{j(>i)} a_{j-} z_{i j}+\frac{1}{2 \sqrt{P}} \sum_{j(\neq i)} a_{j+} x_{i j}$.

Нагадаємо, що змінні $a_{j \pm}$ визначені в (9). Для того щоб факторизувати в (А.4) доданки за індексом частинок $i$, використаємо інтегральну тотожність:

$F\left(\sum_{i} a_{i-} B_{i}\right)=\iint \frac{d \mathbf{u} d \mathbf{v}}{2 \pi} \exp (-i \mathbf{u v}) \exp \left(i \mathbf{v a} \mathbf{a}_{-}\right) F\left(\sum_{i} u_{i} B_{i}\right)$,

де введено позначення: $\mathbf{u v}=\sum_{i} u_{i} v_{i}, \mathbf{v a}_{-}=\sum_{i} v_{i} a_{i-}$. Після нескладних перетворень для $\varphi_{1}(y, z, x)$ отримаємо такий вираз:

$\varphi_{1}(y, z, x)=\int d \mathbf{u} \prod_{j} F_{j}(\mathbf{u}, \mathbf{y}, \mathbf{z}, \mathbf{x})$,

$F_{j}(\mathbf{u}, \mathbf{y}, \mathbf{z}, \mathbf{x})=\frac{1}{2}\left[\exp \left(i L_{j}\right) \cos \left(2 \omega_{j}^{x}\right) \delta\left(u_{j}\right)+\right.$

$+\frac{1}{2} \exp \left(-i L_{j}\right) \exp \left(-2 i \omega_{j}^{z}\right) \delta\left(u_{j}+1 / \sqrt{P}\right)+$

$\left.+\frac{1}{2} \exp \left(-i L_{j}\right) \exp \left(2 i \omega_{j}^{z}\right) \delta\left(u_{j}-1 / \sqrt{P}\right)\right]$,

де позначено

$L_{j}=-\frac{y_{j}}{\sqrt{P}}, \omega_{j}^{z}=\sum_{i(<j)} u_{i} z_{i j}, \omega_{j}^{x}=\sum_{i(\neq j)} u_{i} x_{i j}$.

Формули (А.4) і (А.6) задають точне представлення характеристичної функції. У границі великих $P$ в прийнятій методиці (див. [17]) слід врахувати головні члени розкладу $\varphi_{1}(y, z, x)$ за $1 / P$. Це нескладно зробити розкладаючи на першому кроці функції $\delta\left(u_{j} \pm 1 / \sqrt{P}\right)$ в ряд за степенями $1 / \sqrt{P}$. Зокрема, отримаємо

$F_{j}(\mathbf{u}, \mathbf{y}, \mathbf{z}, \mathbf{x}) \approx F_{j 0}(\mathbf{u}, \mathbf{y}, \mathbf{z}, \mathbf{x})+$

$+\frac{1}{\sqrt{P}} F_{j 1}(\mathbf{u}, \mathbf{y}, \mathbf{z}, \mathbf{x})+\frac{1}{2 P} F_{j 2}(\mathbf{u}, \mathbf{y}, \mathbf{z}, \mathbf{x})$

де

$F_{j 0}(\mathbf{u}, \mathbf{y}, \mathbf{z}, \mathbf{x})=\frac{1}{2}\left[\exp \left(i L_{j}\right) \cos \left(2 \omega_{j}^{x}\right)+\exp \left(-i L_{j}\right) \cos \left(2 \omega_{j}^{z}\right)\right] \delta\left(u_{j}\right)$,

$F_{j 1}(\mathbf{u}, \mathbf{y}, \mathbf{z}, \mathbf{x})=-\frac{1}{2} \exp \left(-i L_{j}\right) \sin \left(2 \omega_{j}^{z}\right) \delta^{\prime}\left(u_{j}\right)$,

$F_{j 2}(\mathbf{u}, \mathbf{y}, \mathbf{z}, \mathbf{x})=\frac{1}{2} \exp \left(-i L_{j}\right) \cos \left(2 \omega_{j}^{z}\right) \delta^{\prime \prime}\left(u_{j}\right)$
Штрихи в (А.9) позначають похідні від $\delta$-функцій. За наявності $\delta$-функцій, після підстановки (А.8) в (А.6), інтеграли за змінними $u_{j}$ обчислюються елементарно. Наступним кроком розкладаємо за степенями $1 / \sqrt{P}$ множники $\exp \left( \pm i L_{j}\right)$. Після виконання вказаних обчислень отримаємо

$\varphi_{1}(y, z, x) \approx 1-\frac{1}{2 P} \sum_{i} y_{i}^{2}-\frac{1}{2 P} \sum_{i<j} z_{i j}^{2}-\frac{1}{2 P} \sum_{i \neq j} x_{i j}^{2}$.

Відповідно для характеристичної функції (А.4) в границі $P \rightarrow$ $\infty$ отримаємо

$\varphi(y, z, x) \approx \exp \left(-\frac{1}{2} \sum_{i} y_{i}^{2}-\frac{1}{2} \sum_{i<j} z_{i j}^{2}-\frac{1}{2} \sum_{i \neq j} x_{i j}^{2}\right)$.

Виконуючи фур'є-перетворення характеристичної функції (А.10), знаходимо асимптотику функції розподілу параметрів гамільтоніана $\Psi(d, J, k)$ :

$\Psi(d, J, k)=\prod_{i} \frac{1}{\sqrt{2 \pi}} \exp \left(-\frac{1}{2} d_{i}^{2}\right) \prod_{i<j} \frac{1}{\sqrt{2 \pi}} \exp \left(-\frac{1}{2} J_{i j}^{2}\right) \times$

$\times \prod_{i \neq j} \frac{1}{\sqrt{2 \pi}} \exp \left(-\frac{1}{2} k_{i j}^{2}\right)$

\section{В. Наближення $1 R S B$}

Наближення $1 R S B$ визначає порушення реплічної симетрії (наближення $R S$ ) на один крок (див., наприклад, [3, 15]). В цьому наближенні матрицю перекриття $\hat{Q}$ задають у вигляді

$Q_{a b}= \begin{cases}Q, & \text { якщо } a=b ; \\ q, & \text { якщо }|a-b|>m ; \\ q_{1}, & \text { якщо }|a-b| \leq m,\end{cases}$

де $m$ визначає кількість реплік у групі при поділі $n$ на $n / m$ груп. Числа $m$ і $n / m$ задаються цілими. 3 означення (В.1) випливає, якщо індекси матриці $Q_{a b}$ належать одній групі, то $Q_{a b}=q$, якщо різним групам, то $Q_{a b}=q_{1}$. Співвідношення (B.1) можна записати також у матричному вигляді

$\hat{Q}=\left(Q-q_{1}\right) \hat{I}_{n}+\left(q_{1}-q\right) \hat{I}_{\frac{n}{m}} \oplus \hat{E}_{m}+q \hat{E}_{n}$.

Матриці $\hat{I}$ і $\hat{E}$ позначають відповідно одиничну матрицю і матрицю, всі елементи якої дорівнюють 1 (індекс вказує порядок матриці). У формулі (В.2) використано також позначення прямого добутку матриць [19]. Використовуючи властивості прямого добутку матриць, нескладно отримати

$\sum_{a, b} Q_{a b}^{2}=n\left\{\left(Q^{2}-q_{1}^{2}\right)+m\left(q_{1}^{2}-q^{2}\right)\right\}$

$\sum_{a, b} \pi_{a} Q_{a b} \pi_{b}=\left(Q-q_{1}\right) \sum_{a} \pi_{a}^{2}+q\left(\sum_{a} \pi_{a}\right)^{2}+$

$+\left(q_{1}-q\right) \sum_{k=1}^{n / m}\left(\sum_{a \in A_{k}} \pi_{a}\right)^{2}$

де $A_{k}$ в (В.4) позначає сукупність реплік, що входять в $k$-у групу. Наступні перетворення є типовими для методу реплік (див. $[3,15])$, тому наведемо їх досить стисло. Отримані вирази (В.3), (В.4) підставляємо у загальні співвідношення (24), (27) і (29). 
Факторизацію за реплічними змінними при обчисленні $Z_{1}(Q)$

(25) виконаємо за допомогою інтегрального перетворення

$e^{\frac{1}{2} x^{2}}=\frac{1}{\sqrt{2 \pi}} \int_{-\infty}^{\infty} d z e^{-\frac{1}{2} z^{2}} e^{z x}$

Після ряду перетворень $Z_{1}(Q)$ запишемо у вигляді

$Z_{1}(Q)=\int_{-\infty}^{\infty} \frac{d z}{\sqrt{2 \pi}} \exp \left(-z^{2} / 2\right) Z_{1}^{n / m}$

де позначено

$Z_{1}=\int_{-\infty}^{\infty} \frac{d z_{1}}{\sqrt{2 \pi}} \exp \left(-z_{1}^{2} / 2\right) \tilde{Z}_{1}^{m}$,

$\tilde{Z}_{1}=\int_{-1}^{1} \frac{d \pi}{2} \exp \left(-\beta \frac{\sqrt{\alpha}}{2} \pi^{2}+\frac{\beta^{2}}{2}\left(Q-q_{1}\right) \pi^{2}\right) \times$

$\times \exp (\beta \sqrt{1+q} \pi z) \exp \left(\beta \sqrt{q_{1}-q} \pi z_{1}\right)$.

Із врахуванням зазначених перетворень, виконуючи граничний перехід $n \rightarrow 0$, для мінімуму (29) отримаємо

$\mathcal{H}=\frac{N}{2}+\frac{N}{4 \sqrt{\alpha}} \lim _{\beta \rightarrow \infty} \beta\left(\left(Q^{2}-q_{1}^{2}\right)+m\left(q_{1}^{2}-q^{2}\right)\right)-$

$-\frac{N}{\sqrt{\alpha}} \frac{1}{m} \lim _{\beta \rightarrow \infty} \frac{1}{\beta} \int_{-\infty}^{\infty} \frac{d z}{\sqrt{2 \pi}} \exp \left(-z^{2} / 2\right) \ln Z_{1}$.

Величина (В.7) залежить від параметрів $Q, q, q_{1}, m$, рівняння для яких можна отримати простим диференціюванням (В.7), а також на основі загального рівняння стаціонарності (27). В результаті нескладних обчислень отримаємо

$Q=\left\langle\frac{\left\langle\left\langle\pi^{2}\right\rangle_{\pi} \tilde{Z}_{1}^{m}\right\rangle_{z_{1}}}{\left\langle\tilde{Z}_{1}^{m}\right\rangle_{z_{1}}}\right\rangle_{z}, \quad q_{1}=\left\langle\frac{\left\langle\langle\pi\rangle_{\pi}^{2} \tilde{Z}_{1}^{m}\right\rangle_{z_{1}}}{\left\langle\tilde{Z}_{1}^{m}\right\rangle_{z_{1}}}\right\rangle_{z}$,

$q=\left\langle\left(\frac{\left\langle\langle\pi\rangle_{\pi} \tilde{Z}_{1}^{m}\right\rangle_{z_{1}}}{\left\langle\tilde{Z}_{1}^{m}\right\rangle_{z_{1}}}\right)^{2}\right\rangle_{z}$.

Усереднення в (В.8) за змінними $z$ i $z_{1}$ здійснюються за такими формулами:

$\langle\ldots\rangle_{z}=\int_{-\infty}^{\infty} \frac{d z}{\sqrt{2 \pi}} \exp \left(-z^{2} / 2\right)(\ldots)$,

$\langle\ldots\rangle z_{1}=\int_{-\infty}^{\infty} \frac{d z_{1}}{\sqrt{2 \pi}} \exp \left(-z_{1}^{2} / 2\right)(\ldots)$,

і також

$\langle\ldots\rangle_{\pi}=\frac{1}{\tilde{Z}_{1}} \int_{-1}^{1} \frac{d \pi}{2} \exp (\beta \varphi(\pi))(\ldots)$,

Прирівнюючи до нуля похідну від (В.7) за параметром $m$, отримуємо рівняння для визначення $m$ :

$\frac{1}{4} \beta\left(q_{1}^{2}-q^{2}\right)+\frac{1}{m^{2}} \frac{1}{\beta}\left\langle\ln Z_{1}\right\rangle_{z}-\frac{1}{m} \frac{1}{\beta}\left\langle\frac{\left\langle\tilde{Z}_{1}^{m} \ln \tilde{Z}_{1}\right\rangle_{z_{1}}}{\left\langle\tilde{Z}_{1}^{m}\right\rangle_{z_{1}}}\right\rangle_{z}=0$

У сукупності формула (В.7) і система рівнянь (В.8), (В.12) дають розв'язок задачі в наближенні $1 R S B$.

3 наведених формул випливає також, що для $q_{1}=q$ наближення $1 R S B$ перейде у наближення симетричних реплік $[3,14]$.

\section{C. Наближення $2 R S B$}

У наближенні $2 R S B$ матрицю перекриття $\hat{Q}$ задають у вигляді $\hat{Q}=\left(Q-q_{2}\right) \hat{I}_{n}+\left(q_{2}-q_{1}\right) \hat{I}_{\frac{n}{m_{2}}} \oplus \hat{E}_{m_{2}}\left(q_{1}-q\right) \hat{I}_{\frac{n}{m_{1}}} \oplus$

$\oplus \hat{E}_{m_{1}}+q \hat{E}_{n}$

де $m_{1}$ визначає кількість реплік у групі при поділі $n$ реплік на $n / m_{1}$ груп, а $m_{2}$ відповідно - кількість реплік в групі при поділі $m_{1}$ реплік на $m_{1} / m_{2}$ груп. При цьому як $m_{1}, m_{2}$ і $m_{1} / m_{2}$ вважаються цілими числами. Зміст решти позначень був вказаний вище. Підставляючи матрицю (С.1) в загальні співвідношення (25), (29), отримуємо

$\sum_{a, b} Q_{a b}^{2}=n\left\{\left(Q^{2}-q_{2}^{2}\right)+m_{2}\left(q_{2}^{2}-q_{1}^{2}\right)+m_{1}\left(q_{1}^{2}-q^{2}\right)\right\}$,

$\sum_{a, b} \pi_{a} Q_{a b} \pi_{b}=\left(Q-q_{2}\right) \sum_{a} \pi_{a}^{2}+\left(q_{2}-q_{1}\right) \sum_{k_{2}=1}^{n / m_{2}}\left(\sum_{a \in A_{k_{2}}} \pi_{a}\right)^{2}+$

$+\left(q_{1}-q\right) \sum_{k_{1}=1}^{n / m_{1}}\left(\sum_{a \in A_{k_{1}}} \pi_{a}\right)^{2}+q\left(\sum_{a} \pi_{a}\right)^{2}$

де $A_{k_{2}}, A_{k_{1}}$ в (С.3) позначають сукупності реплік, що входять у відповідні групи. Як і у випадку $1 R S B$, наступні перетворення полягають у факторизації за реплічними змінними при обчисленні $Z_{1}(Q)(25)$. Після виконання необхідних перетворень для мінімуму (29) отримаємо вираз

$\mathcal{H} / N=\frac{1}{2}+\frac{1}{4 \sqrt{\alpha}} \lim _{\beta \rightarrow \infty} \beta\left(\left(Q^{2}-q_{2}^{2}\right)+m_{2}\left(q_{2}^{2}-q_{1}^{2}\right)+\right.$

$\left.+m_{1}\left(q_{1}^{2}-q^{2}\right)\right)-\frac{1}{\sqrt{\alpha}} \frac{1}{m_{1}} \lim _{\beta \rightarrow \infty} \frac{1}{\beta}\left\langle\ln Z_{1}\right\rangle_{z}$.

Тут введено позначення:

$Z_{1}=\left\langle\left\langle\tilde{Z}_{2}^{m_{2}}\right\rangle_{z_{2}}^{\frac{m_{1}}{m_{2}}}\right\rangle_{z_{1}}, \tilde{Z}_{2}=\int_{-1}^{1} \frac{d \pi}{2} \exp (\beta \varphi(\pi))$,

$\varphi(\pi)=-\frac{\sqrt{\alpha}}{2} \pi^{2}+\frac{\beta}{2}\left(Q-q_{2}\right) \pi^{2}+$

$+\sqrt{q_{2}-q_{1}} \pi z_{2}+\sqrt{q_{1}-q} \pi z_{1}+\sqrt{1+q} \pi z$.

Вільна енергія є функцією параметрів $Q, q_{2}, q_{1}, q, m_{1}, m_{2}$. Рівняння для параметрів $Q, q_{2}, q_{1}, q$, як вже вказувалось вище, отримуємо із загальних рівнянь стаціонарності (27). Зокрема, маємо

$Q=\left\langle\frac{1}{Z_{1}}\left\langle\left\langle\left\langle\pi^{2}\right\rangle \tilde{Z}_{2}^{m_{2}}\right\rangle_{z_{2}}\left\langle\tilde{Z}_{2}^{m_{2}}\right\rangle_{z_{2}}^{\frac{m_{1}}{m_{2}}-1}\right\rangle_{z_{1}}\right\rangle_{z}$,

$q_{2}=\left\langle\frac{1}{Z_{1}}\left\langle\left\langle\langle\pi\rangle^{2} \tilde{Z}_{2}^{m_{2}}\right\rangle_{z_{2}}\left\langle\tilde{Z}_{2}^{m_{2}}\right\rangle_{z_{2}}^{\frac{m_{1}}{m_{2}}-1}\right\rangle_{z_{1}}\right\rangle_{z}$,

$q_{1}=\left\langle\frac{1}{Z_{1}}\left\langle\left\langle\langle\pi\rangle \tilde{Z}_{2}^{m_{2}}\right\rangle_{z_{2}}^{2}\left\langle\tilde{Z}_{2}^{m_{2}}\right\rangle_{z_{2}}^{\frac{m_{1}}{m_{2}}-2}\right\rangle_{z_{1}}\right\rangle_{z}$,

$q=\left\langle\left(\frac{1}{Z_{1}}\left\langle\left\langle\langle\pi\rangle \tilde{Z}_{2}^{m_{2}}\right\rangle_{z_{2}}\left\langle\tilde{Z}_{2}^{m_{2}}\right\rangle_{z_{2}}^{\frac{m_{1}}{m_{2}}-1}\right\rangle_{z_{1}}\right)^{2}\right\rangle_{z}$.

Усереднення за змінними $z, z_{1}, z_{2}$ в формулах (С.4), (С.5) i (C.6) виконуються 3 гауссовими функціями як і в формулі (В.9). Рівняння для параметрів $m_{1}, m_{2}$ отримуємо диференціюванням (C.4) за параметрами $m_{1}$ і $m_{2}$ :

$\frac{\beta}{4}\left(q_{1}^{2}-q^{2}\right)+\frac{1}{\beta m_{1}^{2}}\left\langle\ln \left(Z_{1}\right)\right\rangle_{z}-$ 


$$
\begin{aligned}
& -\frac{1}{\beta m_{1} m_{2}}\left\langle\frac{1}{Z_{1}}\left\langle\left\langle\tilde{Z}_{2}^{m_{2}}\right\rangle_{z_{2}}^{\frac{m_{1}}{m_{2}}} \ln \left\langle\tilde{Z}_{2}^{m_{2}}\right\rangle_{z_{2}}\right\rangle_{z_{1}}\right\rangle_{z}=0, \\
& \frac{\beta}{4}\left(q_{2}^{2}-q_{1}^{2}\right)+\frac{1}{\beta m_{2}^{2}}\left\langle\frac{1}{Z_{1}}\left\langle\left\langle\tilde{Z}_{2}^{m_{2}}\right\rangle_{z_{2}}^{\frac{m_{1}}{m_{2}}} \ln \left\langle\tilde{Z}_{2}^{m_{2}}\right\rangle_{z_{2}}\right\rangle_{z_{1}}\right\rangle_{z}- \\
& -\frac{1}{m_{2}}\left\langle\frac{1}{Z_{1}}\left\langle\left\langle\tilde{Z}_{2}^{m_{2}}\right\rangle_{z_{2}}^{\frac{m_{1}}{m_{2}}-1}\left\langle\tilde{Z}_{2}^{m_{2}} \ln \tilde{Z}_{2}\right\rangle_{z_{2}}\right\rangle_{z_{1}}\right\rangle_{z}=0 .
\end{aligned}
$$

Зауважимо, що всі формули виписані до виконання граничного переходу $\beta \rightarrow \infty$.

Із структури наведеного розв'язку випливає також, що для $q_{2}=q_{1}$ наближення $2 R S B$ перейде у наближення $1 R S B$, а відповідно для $q_{2}=q_{1}$ і $q_{1}=q$ - перейде у наближення $R S$.

1. В. Кинцель, УФН 152, 1 (1987).

2. И.Я. Коренблит, Е.Ф. Шендер, УФН 157, 2 (1989).

3. H. Nishimori, Statistical Physics of Spin Glasses and Information Processing. An Introduction (Clarendon Press, Oxford, 2001).

4. R.N. Mantegna and H.E. Stanley, An Introduction to Econophysics: Correlations and Complexity in Finance (Cambridge Univ. Press, Cambridge, 1999).

5. A. De Martino and M. Marsili, Preprint (physics/ 0606107), (2006).

6. D. Challet, Y. C. Zhang, Physica A 246, 407 (1997).

7. D. Challet, Y. C. Zhang, Physica A 256, 514 (1998).

8. Savit R., Manuca R. and Riolo R., Phys. Rev. Lett. 82, 2203 (1999).

9. D. Challet, M. Marsili and R. Zecchina, Phys. Rev. Lett. 84, 1824 (2000) (cond-mat/9904392).

10. Y.C. Zhang, Physica A 269, 30 (1999), (condmat/9901243).

11. M. Marsili, D. Challet and R. Zecchina, Physica A 280, 522 (2000), (cond-mat/9908480).

12. A. De Martino and M. Marsili, J. Phys. A: Math. Gen. 34, 2525 (2001), (cond-mat/0007397).

13. Э. Мулен, Теория игр с примерами из математической экономики (Мир, Москва, 1972).

14. В. Янішевський, Жуур. фіз. досл. 13, № 2 (2009).

15. M. Mezard, G. Parisi and M.A. Virasoro, Spin Glass Theory and Beyond (World Scientific, Singapore, 1987).

16. Р. Фейнман, Статистическая механика (Мир, Москва, 1975).

17. Феллер В. Введение в теорию вероятностей и ее приложения (Наука, Москва, 1964), Т. 1, 2.

18. J.R.L. de Almeida and D.J. Thouless, J. Phys. A 11, 983 (1978).

19. П. Ланкастер, Теория матрии (Наука, Москва, 1978).

20. A. Crisanti, D.J. Amit, and H. Gutfreund, Europhys. Lett. 2, 337 (1986).
21. Helmut Steffan and Reimer Kühn, (condmat/9404036v1) (1994).

22. S.K. Ghatak and D. Sherrington, J. Phys. C 10, 3149 (1977).

23. G.R. Schreiber, (cond-mat/9612189v2) (1999).

24. F.A. da Costa and J.M de Araújo, cond-mat/0005029v1 (2000).

Одержано 15.02.10

\section{ГАУССОВОЕ ПРИБЛИЖЕНИЕ В ОПТИМИЗАЦИОННОЙ ЗАДАЧЕ МОДЕЛИ ИГРЫ В МЕНЬШИНСТВЕ}

\section{B.С. Янишевский}

$\mathrm{P}$ е $з$ ю м е

Методами статистической физики исследована оптимизационная задача в известной модели игры в меньшинстве. Оптимизационная задача сведена к изучению основного состояния репличного гамильтониана со случайными параметрами некоторой эффективной системы с непрерывным спином. Используя идеи центральных предельных теорем теории вероятности, получены представления для функции распределения параметров гамильтониана и осуществлен переход к гауссовому распределению в случае больших $P$. Используя приближения $1 R S B$ и $2 R S B$ в методе реплик, получена зависимость минимума исследуемой величины от параметра $\alpha$. Показано, что в области применимости предложенный метод дает меньшее значение минимума, чем в оригинальных работах.

\section{GAUSS APPROXIMATION IN THE OPTIMIZATION} PROBLEM OF THE MINORITY GAME MODEL

\section{V.S. Yanishevsky}

Ivan Franko State Drohobych Pedagogical University (24, I. Franko Str., Drohobych 82100, Ukraine)

$\mathrm{S}$ u m m a r y

The optimization problem of the well-known minority game model is studied by methods of statistical physics. The problem is reduced to the study of the ground state of some effective system with continuous spin described by a replica Hamiltonian with random parameters. With the use of the central limit theorem of probability theory, the representations of the distribution function for parameters of the Hamiltonian are obtained, and the transition to the Gauss distribution in the case of large $P$ is realized. Within approximations $1 \mathrm{RSB}$ and $2 \mathrm{RSB}$ in the replica method, the dependence of the minimum of the quantity under study on the parameter $\alpha$ is determined. It is shown that, in the region of applicability, the proposed method gives a less value of the minimum than that obtained in the cited works. 